\title{
LA PERCEPCIÓN DE LA CULTURA EN EL AULA EN FUNCIÓN DE LA VARIABLE GÉNERO EN ALUMNOS Y PROFESORES
}

\author{
Cristina di Giusto Valle \\ Universidad de Burgos \\ cristinadgv@gmail.com \\ Maria Eugenia Martin Palacio \\ Universidad Complutense de Madrid \\ mariaeugeniamartin@edu.ucm.es \\ Bianca Dapelo Pellerano \\ Universidad de Viña del Mar \\ bdapelop@yahoo.com
}

Fecha de Recepción: 30 Enero 2019

Fecha de Admisión: 30 Abril 2019

\section{RESUMEN}

El objetivo de este estudio es conocer la percepción y deseo de cultura emprendedora en profesores y alumnos en función de la variable género. Para ello se sigue el modelo de análisis Cultuala formulado por Martín del Buey de la Universidad de Oviedo y desarrollado por su equipo de investigación, bajo la dirección de la Doctora María Eugenia Martín Palacio de la Universidad Complutense de Madrid. La muestra del profesorado es de 176 participantes de 16 centros educativos. En relación al género, el $66.5 \%$ son profesoras y el $32.4 \%$ profesores. Por otro lado, la muestra de estudiantes está formada por 2308 participantes de 18 centros educativos: 1101 hombres (47.7\%) y 1203 mujeres (52.1\%). La procedencia de la muestra se distribuye en 1922 estudiantes de Asturias (83.3\%), 372 de Castilla y León (16.1\%) y 14 de Cantabria (0.6\%). Los resultados indican que en el profesorado no se han encontrado diferencias estadísticamente significativas en la percepción que tienen de sus desempeños académicos ni en el deseo de una mejora de los mismos. En los alumnos se ha encontrado diferencias tanto en la percepción de los desempeños docentes en el aula como en el deseo de cambio de los mismos por parte de sus profesores. No obstante, en cuanto a la percepción que tienen los alumnos respecto a los aspectos relacionados con la finalidad educativa no existen diferencias de género y tampoco existen en cuanto al deseo de una mejora del escenario educativo. Además, se evidencia que el deseo de mejora de la realidad percibida siempre es superior, y que tanto las medias de profesoras como de alumnas son siempre ligeramente superiores a las de los hombres.

Palabras clave: cultura; educación secundaria; género 


\title{
LA PERCEPCIÓN DE LA CULTURA EN EL AULA EN FUNCIÓN DE LA VARIABLE GÉNERO EN ALUMNOS Y PROFESORES
}

\begin{abstract}
The perception of culture in the classroom according to the gender variable in students and teachers. The objective of this study is to know the perception and desire of entrepreneurial culture in teachers and students according to the gender variable. To do this, we follow the Cultuala analysis model formulated by Martín del Buey of the University of Oviedo and developed by his research team, under the direction of Dr. María Eugenia Martín Palacio of the Complutense University of Madrid. The faculty sample is 176 participants from 16 educational centers. Regarding gender, $66.5 \%$ are women and $32.4 \%$ men. On the other hand, the sample of students consists of 2308 participants from 18 educational centers: 1101 men (47.7\%) and 1203 women (52.1\%). The origin of the sample is distributed in 1922 students from Asturias (83.3\%), 372 from Castilla y León (16.1\%) and 14 from Cantabria $(0.6 \%)$. The results indicate that have not found statistically significant differences in the perception the teachers have of their academic performance or in the desire to improve them. In the students, differences have been found both in the perception of the teaching performances in the classroom and in the desire to change them. However, regarding the perception that the students have regarding the aspects related to the educational purpose, there are no gender differences and neither do they exist in terms of the desire for an improvement of the educational scenario. In addition, it is evident that the desire to improve the perceived reality is always higher, and that both female teachers and female students averages are always slightly higher than those of men.
\end{abstract}

Keyword: culture; secondary education; gender

\section{INTRODUCCIÓN}

Tomando como referencia el modelo de análisis de la Cultura organizacional educativa CULTUAULA formulado por Martín del Buey (1991, 1996, 1997, 1998 y 2003), y desarrollado con posterioridad por su equipo de investigación coordinado por la Dra. Martín Palacio en la Universidad Complutense (Martín Palacio, Fernández y Pizarro, 2010; Martín Palacio, Aguado y Rodríguez, 2013), que ya ha sido presentado en sus líneas básicas en la primera y segunda ponencia de este simposio. En esta ponencia se estudia la percepción que tiene el alumnado y el profesorado en su centro educativo respecto a las creencias en torno a las cuatro dimensiones de análisis propuesta por el modelo cultual, a lo que se añade el estudio de las mejoras posibles que desearía se plasmaran en los desempeños docente de sus profesores. Pero esta percepción y deseo que ha sido estudiado por separado en la segunda y tercera ponencia del simposio se van a estudiar en ésta de forma conjunta en base a la variable género. Se da por contado que existe por parte del profesorado y alumnos su apuesta por los paradigmas cognitivos y humanista pero interesa conocer en qué grado la variable género influye en su grado de implicación y desempeño de estas creencias. En esta línea de investigación se presentan las diferencias existentes en ambos participantes sin cruzar por el momento la percepción de los alumnos en función del género de sus profesores, que será objeto de otro posterior estudio no presente en este simposio.

En la actualidad la variable género ha sido estudiado frecuentemente en el campo educativo, pero en relación a las creencias en torno a los paradigmas educativos no han sido tan frecuentes. Por ello el interés de este estudio en la medida que pueda ser una variable condicionante 0 en su caso determinante que influya en el grado de aceptación del desempeño docente mediatizado por comportamientos pertenecientes a estas creencias. Es constatable que las preguntas que se formuIan en el cuestionario siguen el paradigma cognitivo y humanista de vigente actualidad contraponiéndolo al tradicional y conductual. En consecuencia, los resultados que se obtengan estarán valorando el grado de percepción que tienen los alumnos y profesores del desempeño docente respecto a estos paradigmas y el grado de deseo de cambio, manifestando implícitamente por ello su con- 
formidad a estos paradigmas. De obtener unas puntuaciones bajas se plantearía unas serias dudas respecto a la presencia 0 ausencia en su caso de las creencias implícitas en el profesorado 0 alumnado relacionadas con estos paradigmas. Los datos obtenidos y manifiestos en la segunda y tercera ponencia no indican estos resultados. Claramente se apuesta por una percepción percibida y deseada superior en todos los casos a la media general. Pero no se ofrecen datos en relación a la influencia que tiene el género en las mismas.

De alguna forma cabe preguntarse en qué medida los alumnos y los profesores se diferencias de las alumnas y profesoras en la percepción y en el deseo de mejora de esa percepción en sus desempeños docentes. Esto de alguna manera estaría explicitando el grado de apuesta que condiciona el género en estos desempeños y si la apuesta es mayor en unos sobre otros, si la diferencia es importante y significativa y si se da en todos los aspectos o consideraciones en que lo estudia el modelo de análisis que se asume en esta investigación. Esta ponencia se centra únicamente en el estudio de consonancia y disonancias entre las cultura percibida y deseada en función del género. Otras variables como la edad y el centro pueden ser estudiadas. En concreto la del centro es objeto de otra comunicación en este simposio. Pero la variable edad que también ha sido estudiado no se expone en el mismo (Arnaiz, 2015). Lo mismo ocurre respecto a la variable de ramas educativas (Arnaiz, Di Giusto y Martín del Buey (2014) y en otros ámbitos como son los universitarios (Arnaiz, Pizarro y Martín del Buey (2012).

\section{METODOLOGÍA}

\section{Participantes}

La muestra de profesorado estuvo formada por 176 participantes de 16 centros educativos.

En relación al género, los docentes que participaron en este estudio fueron en su gran mayoría mujeres; $66.5 \%$ profesoras frente al $32.4 \%$ de los profesores (Tabla 1 )

Tabla 1

Frecuencia de género del profesorado

\begin{tabular}{lcc}
\hline & Frecuencia & Porcentaje \\
\hline Hombre & 57 & 32.4 \\
Mujer & 117 & 66.5 \\
Perdidos & 2 & 1.1 \\
Total & 176 & 100.0 \\
\hline
\end{tabular}

Como se ve en la Tabla 2, la muestra estuvo formada por 133 docentes de Asturias (75.6\%), 42 de Castilla y León $(23.9 \%$ ) y uno de Cantabria $(0.6 \%)$.

Tabla 2

Frecuencia de Comunidad Autónoma del profesorado

\begin{tabular}{lcc}
\hline & Frecuencia & Porcentaje \\
\hline Asturias & 133 & 75.6 \\
Castilla y León & 42 & 23.9 \\
Cantabria & 1 & .6 \\
Total & 176 & 100.0 \\
\hline
\end{tabular}




\section{LA PERCEPCIÓN DE LA CULTURA EN EL AULA EN FUNCIÓN DE LA VARIABLE GÉNERO EN ALUMNOS Y PROFESORES}

La muestra de estudiantes estuvo formada por 2308 participantes de 18 centros educativos.

Los estudiantes participantes de este estudio eran 1101 hombres (47.7\%) y 1203 mujeres $(52.1 \%)$, encontrándose 4 personas que no indicaron su género $(0.2 \%)$, tal y como se muestra en la Tabla 3.

Tabla 3

Frecuencia de género del alumnado

\begin{tabular}{lcc} 
& Frecuencia & Porcentaje \\
\hline Hombre & 1101 & 47.7 \\
Mujer & 1203 & 52.1 \\
Perdidos & 4 & 0.2 \\
Total & 2308 & 100.0 \\
\hline
\end{tabular}

En cuanto a la procedencia del alumnado, la muestra estuvo formada por 1922 estudiantes de Asturias (83.3\%), 372 de Castilla y León (16.1\%) y 14 de Cantabria (0.6\%), Tabla 4.

Tabla 4

Frecuencia de Comunidad Autónoma del alumnado

\begin{tabular}{lcc}
\hline & Frecuencia & Porcentaje \\
\hline Asturias & 1922 & 83.3 \\
Castilla y León & 372 & 16.1 \\
Cantabria & 14 & .6 \\
Total & 2308 & 100.0 \\
\hline
\end{tabular}

\section{Cuestionario}

Cuestionario Cultuala de Arnaiz (2015), evalúa la cultura emprendedora percibida y deseada para profesores y alumnos.

El cuestionario de cultura emprendedora percibida en el alumnado tiene una fiabilidad de .916; y el de cultura emprendedora percibida en el profesorado es de .887.

El cuestionario de cultura emprendedora deseada en el alumnado tiene una fiabilidad de .888 y el de cultura emprendedora deseada en el profesorado es de .919

En relación a la validez de estructura se ha obtenido una estructura factorial afín a la planteada en torno a la presencia de cuatro dimensiones o factores, la cual a su vez fue confirmada mediante un Análisis Factorial Confirmatorio.

\section{Recogida de datos}

La aplicación de los cuestionarios tuvo lugar en el aula habitual del alumnado dentro del horario escolar. Se realizó de forma colectiva, con una duración aproximada de 15 minutos. En el profesorado fue durante la aplicación de la prueba en los alumnos 0 en la sala de profesores. Los cuestionarios fueron administrados por personal entrenado para ello y se aseguró el anonimato y la confidencialidad de los datos. Estos datos han sido recogidos y valorados por Arnaiz (2015).

\section{Procesamiento}

Para el análisis de los datos del estudio se empleó el paquete estadístico IBM SPSS Statistics 18.0, el AMOS 18. Para el estudio de diferencias, globales, por factores, y en función del género, se utilizaron las pruebas ANOVA. 


\section{Resultados}

En la Tabla 5 se presentan los estadísticos de alumnos y alumnas en los cuatro factores: Finalidad educativa, Proceso de enseñanza; Proceso de aprendizaje; y escenario educativo.

Tabla 5

Estadísticos descriptivos según género del alumnado

\begin{tabular}{llcrc}
\hline & Género & Media & Desv. Típ. & N \\
\hline \multirow{3}{*}{ Finalidad percibida } & Hombre & 18,5722 & 4,84407 & 1101 \\
& Mujer & 18,8421 & 4,57803 & 1203 \\
& Total & 18,7131 & 4,70794 & 2304 \\
\hline \multirow{3}{*}{ Proceso de enseñanza percibida } & Hombre & 18,6866 & 4,33012 & 1101 \\
& Mujer & 19,0881 & 4,01502 & 1203 \\
& Total & 18,8963 & 4,17248 & 2304 \\
\hline \multirow{3}{*}{ Proceso de aprendizaje percibida } & Hombre & 20,0254 & 4,43532 & 1101 \\
& Mujer & 20,5328 & 4,22254 & 1203 \\
& Total & 20,2904 & 4,33201 & 2304 \\
\hline \multirow{3}{*}{ Escenario percibido } & Hombre & 19,4896 & 4,38542 & 1101 \\
& Mujer & 19,8928 & 4,04315 & 1203 \\
& Total & 19,7001 & 4,21408 & 2304 \\
\hline \multirow{3}{*}{ Finalidad deseada } & Hombre & 24,5431 & 3,97186 & 1101 \\
& Mujer & 25,2078 & 3,69895 & 1203 \\
& Total & 24,8902 & 3,84532 & 2304 \\
\hline \multirow{3}{*}{ Proceso de enseñanza deseada } & Hombre & 24,4096 & 3,79668 & 1101 \\
& Mujer & 24,9701 & 3,32851 & 1203 \\
& Total & 24,7023 & 3,57014 & 2304 \\
\hline \multirow{2}{*}{ Proceso de aprendizaje deseada } & Hombre & 24,7684 & 3,81480 & 1101 \\
& Mujer & 25,1072 & 3,43385 & 1203 \\
& Total & 24,9453 & 3,62406 & 2304 \\
\hline \multirow{2}{*}{ Escenario deseada } & Hombre & 25,8065 & 3,60931 & 1101 \\
& Mujer & 25,9701 & 3,46181 & 1203 \\
& Total & 25,8919 & 3,53324 & 2304 \\
\hline \multirow{2}{*}{ Cultura emprendedora percibida } & Hombre & 76,7738 & 16,07564 & 1101 \\
& Mujer & 78,3558 & 14,94432 & 1203 \\
& Total & 77,5998 & 15,51200 & 2304 \\
\hline & Hombre & 99,5277 & 13,11690 & 1101 \\
& Mujer & 101,2552 & 11,75005 & 1203 \\
& Total & 100,4297 & 12,44923 & 2304 \\
\hline & & & & \\
& & &
\end{tabular}

La Traza de Pillai sobre estos datos resultó estadísticamente significativo ( $F=4.422, p=.000$, $\eta 2=$ .015), aunque con un tamaño de efecto bajo.

A continuación se efectuaron ANOVAS para cada una de las variables dependientes, cuyos resultados se presentan en la Tabla 6. 


\section{LA PERCEPCIÓN DE LA CULTURA EN EL AULA EN FUNCIÓN DE LA VARIABLE GÉNERO EN ALUMNOS Y PROFESORES}

Tabla 6

ANOVA para los escalas en función de la variable género en el alumnado

\begin{tabular}{lrcc}
\hline Variable dependiente & \multicolumn{1}{c}{ F } & Significación & Eta al cuadrado parcial \\
\hline Finalidad percibida & 1,889 &, 169 &, 001 \\
Proceso enseñanza percibida & 5,332 &, 021 &, 002 \\
Proceso Aprendizaje percibida & 7,910 &, 005 &, 003 \\
Escenario percibido & 5,273 &, 022 &, 002 \\
Finalidad deseada & 17,297 &, 000 &, 007 \\
Proceso Enseñanza deseada & 14,248 &, 000 &, 006 \\
Proceso Aprendizaje deseada & 5,034 &, 025 &, 002 \\
Escenario deseado & 1,232 &, 267 &, 001 \\
Cultura emprendedora percibida & 5,992 &, 014 &, 003 \\
Cultura emprendedora deseada & 11,118 &, 001 &, 005 \\
\hline
\end{tabular}

Los resultados expuestos indican la existencia de diferencias estadísticamente significativas en todas las escalas del cuestionario, a excepción de Finalidad en cultura emprendedora percibida y Escenario en cultura emprendedora deseada.

En la Tabla 7 se presentan los estadísticos descriptivos de profesores y profesoras en los cuatro factores, tanto a nivel de percibida como de deseada.

Tabla 7

Estadísticos descriptivos según género del profesorado

\begin{tabular}{llccc}
\hline & Género & Media & Desv. Típ. & N \\
\hline \multirow{2}{*}{ Finalidad percibida } & Hombre & 24,0000 & 3,80319 & 57 \\
& Mujer & 23,8718 & 3,43797 & 117 \\
& Total & 23,9138 & 3,55120 & 174 \\
\hline \multirow{2}{*}{ Proceso de enseñanza percibida } & Hombre & 23,4912 & 3,47503 & 57 \\
& Mujer & 22,9145 & 3,23391 & 117 \\
& Total & 23,1034 & 3,31587 & 174 \\
\hline \multirow{2}{*}{ Proceso de aprendizaje percibida } & Hombre & 24,6842 & 3,81331 & 57 \\
& Mujer & 24,5214 & 3,17479 & 117 \\
& Total & 24,5747 & 3,38693 & 174 \\
\hline \multirow{2}{*}{ Escenario percibido } & Hombre & 22,7544 & 3,90641 & 57 \\
& Mujer & 22,1368 & 3,14288 & 117 \\
& Total & 22,3391 & 3,41282 & 174 \\
\hline \multirow{2}{*}{ Finalidad deseada } & Hombre & 27,2632 & 3,58333 & 57 \\
& Mujer & 27,6239 & 2,61536 & 117 \\
& Total & 27,5057 & 2,96170 & 174 \\
\hline \multirow{2}{*}{ Proceso de enseñanza percibida } & Hombre & 27,4561 & 2,96449 & 57 \\
& Mujer & 27,5043 & 2,79354 & 117 \\
& Total & 27,4885 & 2,84216 & 174 \\
\hline \multirow{2}{*}{ Proceso de aprendizaje percibida } & Hombre & 27,2281 & 3,22943 & 57 \\
& Mujer & 27,4444 & 2,68599 & 117 \\
& Total & 27,3736 & 2,86772 & 174 \\
\hline \multirow{2}{*}{ Escenario percibido } & Hombre & 25,9474 & 3,29730 & 57 \\
& Mujer & 26,1966 & 2,98051 & 117 \\
& Total & 26,1149 & 3,08052 & 174 \\
\hline \multirow{2}{*}{ Cultura emprendedora percibida } & Hombre & 94,9298 & 12,87559 & 57 \\
& Mujer & 93,4444 & 10,86948 & 117 \\
& Total & 93,9310 & 11,54863 & 174 \\
\hline \multirow{2}{*}{ Cultura emprendedora deseada } & Hombre & 107,8947 & 11,92938 & 57 \\
& Mujer & 108,7692 & 9,96146 & 117 \\
& Total & 108,4828 & 10,61939 & 174 \\
\hline
\end{tabular}


La Traza de Pillai sobre estos datos resultó estadísticamente no significativo ( $F=.655, p=.730$, $\eta 2=.031$ ), por lo que no existen diferencias significativas entre las profesoras y profesores en ninguno de los factores del constructo Cultuaula.

En la Tabla 8 se presentan los estadísticos de docentes y estudiantes en los distintos factores: Finalidad educativa; Proceso de Enseñanza; Proceso de Aprendizaje y Escenario educativo, tanto para la cultura emprendedora percibida como para la deseada.

Tabla 8

Estadísticos descriptivos alumnado y profesorado

\begin{tabular}{llccc}
\hline & Profesión & Media & Desv. Típ. & $\mathrm{N}$ \\
\hline \multirow{3}{*}{ Finalidad percibida } & Alumnado & 18,7158 & 4,70553 & 2308 \\
& Profesorado & 23,9091 & 3,53153 & 176 \\
& Total & 19,0837 & 4,81952 & 2484 \\
\hline \multirow{3}{*}{ Proceso Enseñanza percibida } & Alumnado & 18,9021 & 4,17185 & 2308 \\
& Profesorado & 23,1080 & 3,29757 & 176 \\
& Total & 19,2001 & 4,25466 & 2484 \\
\hline \multirow{3}{*}{ Proceso Aprendizaje percibida } & Alumnado & 20,2968 & 4,33110 & 2308 \\
& Profesorado & 24,5568 & 3,37167 & 176 \\
& Total & 20,5986 & 4,40741 & 2484 \\
\hline \multirow{3}{*}{ Escenario percibido } & Alumnado & 19,7062 & 4,21326 & 2308 \\
& Profesorado & 22,3466 & 3,40072 & 176 \\
& Total & 19,8933 & 4,21515 & 2484 \\
\hline \multirow{3}{*}{ Finalidad deseada } & Alumnado & 24,8947 & 3,84380 & 2308 \\
& Profesorado & 27,5170 & 2,94710 & 176 \\
& Total & 25,0805 & 3,84611 & 2484 \\
\hline \multirow{3}{*}{ Proceso Enseñanza deseada } & Alumnado & 24,7075 & 3,57116 & 2308 \\
& Profesorado & 27,4943 & 2,83448 & 176 \\
& Total & 24,9050 & 3,59540 & 2484 \\
\hline \multirow{2}{*}{ Proceso Aprendizaje deseada } & Alumnado & 24,9502 & 3,62475 & 2308 \\
& Profesorado & 27,3807 & 2,85206 & 176 \\
& Total & 25,1224 & 3,62903 & 2484 \\
\hline \multirow{2}{*}{ Escenario deseado } & Alumnado & 25,8977 & 3,53335 & 2308 \\
& Profesorado & 26,0909 & 3,07297 & 176 \\
& Total & 25,9114 & 3,50252 & 2484 \\
\hline \multirow{2}{*}{ Cultura emprendedora percibida } & Alumnado & 77,6209 & 15,50725 & 2308 \\
& Profesorado & 93,9205 & 11,48736 & 176 \\
& Total & 78,7758 & 15,81857 & 2484 \\
\hline & Alumnado & 100,4502 & 12,44983 & 2308 \\
& Profesorado & 108,4830 & 10,56191 & 176 \\
& Total & 101,0193 & 12,49495 & 2484 \\
\hline & & & & \\
& & & \\
& & & & \\
& & & & \\
& & &
\end{tabular}

La Traza de Pillai sobre estos datos resultó estadísticamente significativo ( $F=47.451, p=.000$, $\eta 2=.133)$.

A continuación se efectuaron ANOVAS para cada una de las variables dependientes, cuyos resultados se presentan en la Tabla 9, teniendo en cuenta la profesión, es decir, si son estudiantes 0 docentes. 


\section{LA PERCEPCIÓN DE LA CULTURA EN EL AULA EN FUNCIÓN DE LA VARIABLE GÉNERO EN ALUMNOS Y PROFESORES}

Tabla 9

ANOVA para las escalas en función de la variable Profesión

\begin{tabular}{|c|c|c|c|c|}
\hline & Variable dependiente & $\mathrm{F}$ & Significación & $\begin{array}{l}\text { Eta al cuadrado } \\
\text { parcial }\end{array}$ \\
\hline \multirow{10}{*}{ Profesión } & Finalidad percibida & 205,520 &, 000 &, 076 \\
\hline & $\begin{array}{l}\text { Proceso Enseñanza } \\
\text { percibida }\end{array}$ & 170,724 &, 000 &, 064 \\
\hline & $\begin{array}{l}\text { Proceso Aprendizaje } \\
\text { percibida }\end{array}$ & 162,727 &, 000 &, 062 \\
\hline & Escenario percibido & 65,840 &, 000 & ,026 \\
\hline & Finalidad deseada & 78,390 &, 000 & 031 \\
\hline & Proceso Enseñanza deseada & 102,250 &, 000 &, 040 \\
\hline & $\begin{array}{l}\text { Proceso Aprendizaje } \\
\text { deseada }\end{array}$ & 75,554 &, 000 &, 030 \\
\hline & Escenario deseado & ,497 & ,481 &, 000 \\
\hline & $\begin{array}{l}\text { Cultura emprendedora } \\
\text { percibida }\end{array}$ & 186,605 &, 000 & 070 \\
\hline & $\begin{array}{l}\text { Cultura emprendedora } \\
\text { deseada }\end{array}$ & 69,450 &, 000 &, 027 \\
\hline
\end{tabular}

Los resultados expuestos indican la existencia de diferencias estadísticamente significativas en todas las escalas del cuestionario a excepción del factor Escenario en cultura emprendedora percibida.

\section{Discusión y conclusiones}

Respecto a los alumnos (varones) la valoración que hacen con carácter general del desempeño de la actividad docente en el aula en función de los criterios utilizados para el análisis de las creencias se encuentra en torno al 76 frente a una puntuación máxima de 120 puntos. En esta misma línea se encuentra las puntuaciones medias de las alumnas que siempre son algo superiores a las obtenidas por sus compañeros pero dentro de esos mismos límites. En relación a la puntuación total de percepción de la cultura su puntuación media es de 78 sobre una puntuación máxima de 120 puntos. Las puntuaciones medias de los alumnos en relación a cada uno de los aspectos que se contempla en el modelo Cultuaula se encuentran entre el 18, 19 y 20 sobre un total de 30 puntos siendo la puntuación de la percepción de los procesos de aprendizaje la de 20 y la más baja la referida a la percepción de la finalidad educativa. Existen diferencias en esa percepción a favor de las alumnas. Son pequeñas pero son estadísticamente significativas a excepción del nivel de percepción que tiene de la finalidad, cuyas diferencias no lo son. También son significativas las diferencias de medias respecto a la totalidad.

Una lectura de estas diferencias se puede plasmar en los siguientes aspectos:

En lo referente a la finalidad conviene recordar que los alumnos no presentan diferencias de género y valoran el grado en que sus profesores trabajan la autoestima de sus alumnos, su capacidad de resolución de problemas, el análisis de las notas obtenidas, el desarrollo de habilidades de comunicación y la creatividad.

En cambio sí que hay diferencias de género respecto a los procesos de enseñanza. Ellas valoran con mayor intensidad el grado en que el profesorado enseña a buscar información en distintas fuentes (libros, Internet, periódicos...), que en las notas también se tengan en cuenta todas las acti- 
vidades que se han realizado, que proponga diferentes actividades acerca de lo que explica en clase, por ejemplo, excursiones, proyectos de investigación, debates, exposiciones, etc., que relacione lo que explica en clase con lo que se enseña en otras asignaturas o con temas que el alumnado ya conoce y que motive al alumnado a proponer actividades para trabajar los temas que se explican en clase. Esta puntuación nuevamente en términos relativos no es baja. Indica que el centro se trabaja en la línea del paradigma cognitivo y humanista, pero todavía con algunas carencias.

Y estas diferencias de género también se aprecia en lo referente a la puntuación obtenida respecto a los procesos de aprendizaje que desarrollan sus profesores consistentes en que se las motive a relacionar situaciones de la vida real con lo explicado en clase, se insista más en la comprensión de lo que se enseña que en la memorización, se valore los trabajos en grupo, se las anime a exponer trabajos a sus compañeros, se las motive a expresar con sus propias palabras lo que se explica en clase y se las oriente sobre cómo preparar su asignatura,

Finalmente la media obtenida respecto a la valoración del escenario en donde se desarrolla la actividad docente es mayor la de las alumnas pues estás valoran con mayor intensidad aspectos tan variados como la colocación de los muebles en las aulas de tal forma que se las permita una buena comunicación entre todos los que están en clase (por ejemplo con las mesas colocadas en forma de $U$ ) y que ello permita una buena relación entre todos, que haya espacios adaptados para hacer actividades propuestas por el profesorado como por ejemplo: trabajos con ordenadores, visualización de películas, trabajos en grupos, etc. que en las clases se utilicen nuevas tecnologías como por ejemplo: pizarras digitales, ordenadores, webs... y que se deje tiempo para preparar otras asignaturas. Nuevamente la puntuación media no es baja en términos relativos.

En cuanto al deseo de cambio de esa percepción en relación a cada uno de los aspectos que se contempla en el modelo cultuaula en los alumnos (varones) sus puntuaciones se encuentran en torno a los 24 y 25 puntos sobre una puntuación total de 30 indicando con ello un deseo sustancialmente significativo de mejora en los desempeños docentes. La puntuación total en la escala de deseo se encuentra en torno 99 puntos sobre una puntuación máxima de 120 puntos. En esa misma línea de deseo de una cultura emprendedora en los desempeños docentes se encuentran las alumnas cuyas puntuaciones dos del 24 y 25 sobre un total máximo de 30 puntos. A nivel general su deseo gira en torno a 101 sobre un total máximo de 120 puntos. También lo son significativas las diferencias de medias respecto a la totalidad. Estas diferencias en el deseo de una mejora en los desempeños docentes en función del género son siempre a favor de las alumnas, aunque pequeñas, son estadísticamente significativas a excepción del nivel de percepción que tiene del escenario cuyas diferencias no lo son.

En relación a las puntuaciones medias obtenidas en el profesorado nos encontramos que si bien no existen diferencias significativas entre los varones y las mujeres tanto en los aspectos de percepción y deseo merece la pena destacar que en cuanto a la percepción de la cultura en las aulas las puntuaciones de los profesores son algo más altas de las obtenidas por sus compañeras y esto se invierte a la hora del deseo de un cambio de desempeño de esas culturas, donde ellas siempre obtienen puntuaciones más altas.

Cabe preguntarse si es posible deducir que estas diferencias de género en la percepción y en el deseo de mejora de quehacer docente a favor de las alumnas implica una mayor apuesta de estas por los paradigmas cognitivo y humanístico, pero que esta desaparece con la edad dado que entre el profesorado estas diferencias no aparecen de forma estadísticamente significativa.

Finalmente cabe también señalar que la percepción que tienen los profesores en general de los desempeños docentes son siempre más altas que las que arrojan lo alumnos. Y esto siempre en relación a la percepción y al deseo. 


\section{LA PERCEPCIÓN DE LA CULTURA EN EL AULA EN FUNCIÓN DE LA VARIABLE GÉNERO EN ALUMNOS Y PROFESORES}

\section{CONCLUSIONES}

A modo de conclusión se han diferencias significativas en función del género del alumnado tanto en la cultura emprendedora percibida como en la cultura emprendedora deseada Por otro lado, no existen diferencias en función del género entre la cultura percibida y la cultura deseada en las aulas por parte de los profesores, ni a nivel global ni en relación a la finalidad educativa, procesos de enseñanza, procesos de aprendizaje y escenario educativo Se evidencia que el deseo de mejora de la realidad percibida siempre es superior y que tanto las profesoras como las alumnas sus medias son siempre ligeramente superior a los hombres aunque estadísticamente en el caso de las profesoras estas diferencias no sea significativas.

\section{REFERENCIAS BIBLIOGRÁFICAS}

Arnaiz, A. (2015). La cultura emprendedora en la educación secundaria. Tesis doctoral defendida en la Universidad de Oviedo. . Universidad de Oviedo.

Arnaiz, A., Di Giusto, C. y Zamorano, M. (2014). Cultura organizaiconal en la Universidad de Burgos. Estudio diferencia por ramas educativas. Actas del Congreso Internacioanl Docencia Universitaria e Innovación. Tarragona.

Arnaiz, A., Pizarro, J.P. y Martín del Buey, F. (2012). Análisis de la cultura organizacional percibida y paradigma por alumnos en contextos universitarios: estudio piloto de la facultad de psicología de la Universidad de Oviedo. Actas de VII congreso Iberoamericano de Docencia Universitaria. Faculdade de Psicologia e de Ciências da Educação da Universidade do Porto, Porto, Portugal.

Martín del Buey, F. (1991). Modelo de análisis de presunciones culturales en el aula. Actas del I Congreso Internacional de Psicología y educación (pp. 168-170). Madrid.

Martín del Buey, F. (1996). El rol del profesor en el umbral del siglo XXI. Revista Magister, 14, 205215.

Martín del Buey, F. (1997). Modelos implícitos en la reforma educativa. Revista Magister, 15, 90-97.

Martín del Buey, F. (1998). Psicología de las culturas. Actas del Congreso internacional sobre el discurso artístico Norte y Sur. Volumen II, (pp. 415-438). Oviedo.

Martín del Buey, F. (2003). La cultura del chip en el aula: del conflicto a la seducción sin perder identidad. X Congreso internacional sobre el discurso artístico: "El discurso visual". Volumen II, (pp. 81-97). Oviedo.

Martín Palacio, M.E., Fernández, A. y Pizarro, J.P. (2010). De la cultura del espectáculo al saber académico sin perecer en el intento: una experiencia de empleo de la producción fílmica en la universidad. Internacional Journal of Developmental and Educational Psychology (INFAD Revista de Psicología. Psicología Positiva, nuevas tecnologías y realidad actual), 4, 229-236.

Martín Palacio, M.E., Aguado, J.J. y Rodríguez, E. (2013). Cultura percibida y deseada en contextos educativos universitarios. En J.J. Gázquez Linares, M.C. Pérez Fuentes y M.M. Molero Jurado (Comps.), La Convivencia Escolar: Un acercamiento multidisciplinar (pp. 69-74). Almería: ASUNIVEP. 